\title{
Erratum to: Perfusion index and left ventricular output correlation in healthy term infants
}

\author{
Iuri Corsini $^{1}$ - Alessandra Cecchi ${ }^{1} \cdot$ Caterina Coviello $^{1} \cdot$ Carlo Dani $^{1,2}$
}

Published online: 5 June 2017

(C) Springer-Verlag Berlin Heidelberg 2017

\section{Erratum to: Eur J Pediatr}

DOI 10.1007/s00431-017-2920-1

The original version of this article, unfortunately, contained errors. The published version of the manuscript was not the revised version thus the changes were made. The original article was corrected.

The online version of the original article can be found at http://dx.doi.org/ 10.1007/s00431-017-2920-1

Carlo Dani

cdani@unifi.it

Iuri Corsini

corsiniiuri@gmail.com

Alessandra Cecchi

ale.cecchi76@gmail.com

Caterina Coviello

caterinacoviello83@yahoo.it

1 Division of Neonatology, Careggi University Hospital, University of Florence School of Medicine, Florence, Italy

2 Department of Neurosciences, Psychology, Drug Research and Child Health, Careggi University Hospital of Florence, Florence, Italy 